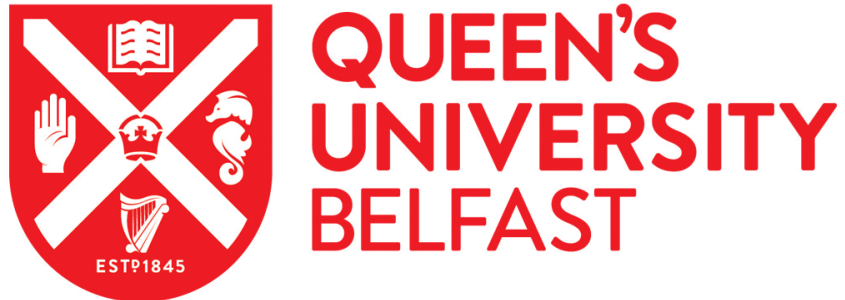

\section{Diabetes Distress: Understanding the Hidden Struggles of Living with Diabetes and Exploring Intervention Strategies}

Berry, E., Lockhart, S., Davies, M., Lindsay, J., \& Dempster, M. (2015). Diabetes Distress: Understanding the Hidden Struggles of Living with Diabetes and Exploring Intervention Strategies. Postgraduate Medical Journal, 91(1075), 278-283. https://doi.org/10.1136/postgradmedj-2014-133017

Published in:

Postgraduate Medical Journal

Document Version:

Peer reviewed version

Queen's University Belfast - Research Portal:

Link to publication record in Queen's University Belfast Research Portal

\section{Publisher rights}

(C) 2015 BMJ Publishing Group

This article has been accepted for publication in Postgraduate Medical Journal following peer review. The definitive copy edited, typeset version is available online at: www.postgradmedj.com

Berry, E, Lockhart, S, Davies, M, Lindsay, J \& Dempster, M 2015, 'Diabetes Distress: Understanding the Hidden Struggles of Living with Diabetes and Exploring Intervention Strategies' Postgraduate Medical Journal, vol 91, no. 1075, pp. 278-283., 10.1136/postgradmedj-2014133017

General rights

Copyright for the publications made accessible via the Queen's University Belfast Research Portal is retained by the author(s) and / or other copyright owners and it is a condition of accessing these publications that users recognise and abide by the legal requirements associated with these rights.

Take down policy

The Research Portal is Queen's institutional repository that provides access to Queen's research output. Every effort has been made to ensure that content in the Research Portal does not infringe any person's rights, or applicable UK laws. If you discover content in the Research Portal that you believe breaches copyright or violates any law, please contact openaccess@qub.ac.uk. 


\title{
Diabetes Distress: Understanding the Hidden Struggles of Living with Diabetes and Exploring Intervention Strategies.
}

\begin{abstract}
Diabetes Distress is a rational emotional response to the threat of a life-changing illness. Distinct from depression, it is rooted in the demands of diabetes management and is a product of psychological adjustment. Diabetes distress has been found to be significantly associated with $\mathrm{HbA}_{1 \mathrm{c}}$ and self-care, which demonstrates its clinical use in treatment outcomes. Interpersonal factors such as perceived support and protectiveness of partners significantly contribute to elevated distress, suggesting that these are valued areas of focus for interventions. Pioneering large-scale research, DAWN2, gives voices to the families of those with diabetes and reaffirms the need to consider psychosocial factors in routine diabetes care. Structured diabetes education programmes are the most widely used in helping individuals cope with diabetes, but they fail to consider the psychological or interpersonal aspects of diabetes management. Psycho-educational approaches are found to be effective in reducing diabetes distress while also improving $\mathrm{HbA}_{1 \mathrm{c}}$. Certain limitations in the current literature are discussed, along with future directions. Of utmost importance is the need for health practitioners, irrespective of background, to demonstrate an understanding of diabetes distress and actively engage in discussion with individuals struggling to cope with diabetes; to normalize this and integrate it into routine diabetes practice.
\end{abstract}

Key Search Terms: Diabetes, diabetes distress, perceived support, social support, coping behaviours, diabetes and depression, structured diabetes education, diabetes distress interventions, illness perceptions [MEDLINE, PSYCHINFO].

\section{Introduction}

Diabetes affects not only an individual's physical health, but it can also have a profound impact on mental wellbeing. For the majority of individuals, a diagnosis of diabetes involves acquiring a demanding subset of added responsibilities, planning and self-monitoring in order to effectively manage their illness. Such a process requires marked re-adjustment of daily life, which can be physically and psychologically draining, and which inevitably permeates through an individual's social environment, [1]. With the incidence of Type 1 [2] and Type 2 [3] diabetes increasing, diabetes has become a focal point of concern in health research, particularly in relation to the psychological, social and behavioural factors implicated in effective illness management. The recognition and understanding of emotional issues in diabetes care is a crucial step towards endorsing support which is not simply prescriptive, but is person-centred and collaborative. For instance by empowering people with diabetes to be as much involved in their self-care plan as possible in the early phases of diagnosis, [4]. Developing a more coherent understanding of the broader psychosocial issues in the context of diabetes management may also inform the design of an effective, holistic intervention for individuals struggling to cope with diabetes. Addressing the factors underpinning maladaptive coping behaviours may in turn prevent physical deterioration and further medical complications.

Attention to date has been largely focused on the role of depression in diabetes. A systematic review has shown depression to be 2-3 times as common in individuals with diabetes compared to people who do not have diabetes, $[5,6]$ which has aroused the attention of health practitioners in the UK. The Quality and Outcomes Framework (QOF) has recommended two indicatory questions drawn from the Patient Health Questionnaire-2 (PHQ-2) as a measure of depressive symptoms over the past two 
weeks, [7]. Though, evidence speculates that this simplistic measure has resulted in a disproportionately high diagnosis of depression in people with diabetes [8]. Screening for depression is undoubtedly a valuable aspect of health care, and while this is important in a clinical context, it is perhaps insufficient to solely focus on this definition in the context of mental health in diabetes. Fisher and colleagues have shown that, in a large sample of people with diabetes, $70 \%$ of those identified as having depression by the Centre of Epidemiological Studies Depression scale (CESD) are not clinically depressed, as determined when using the gold standard clinical assessment; the Composite International Diagnostic Interview score (CIDI), [9]. The incongruity in diagnoses suggests that a different, more specific form of distress may be present in individuals with diabetes who are not clinically depressed but are exhibiting evident psychological difficulties,[9].

This review intends to provide a coherent account of diabetes distress in definition and aetiology and will explore how this might impact on diabetes management and overall wellbeing. Our main objectives are: to raise the awareness of diabetes distress with health practitioners of all backgrounds, to encourage informal discussion of certain focal aspects of distress with individuals with diabetes in appointments and groups (as well as outlining existing formal intervention strategies), and finally to identify where the literature should go next if progress is to made in reducing emotional distress in diabetes.

\section{Defining Diabetes Distress: More than Just Depression}

Diabetes distress is a form of emotional distress which is specific to diabetes. Conceptually rooted in coping and appraisal models in the context of illness, it is defined as a psychological reaction to the threat of diabetes i.e. when an individual diagnosed with diabetes considers the coping resources they possess as insufficient to manage the illness threat, thus triggering emotional distress specific to the diabetes [10]. Diabetes distress has been defined across domains relating to: Diabetic regimen i.e. the monitoring of blood glucose, dietary control, increasing physical activity; interpersonal or relational issues, referring to conflicts with carers, partners and friends; and emotional burden i.e. feeling demotivated, unsupported emotionally, misunderstood and worrying about future complications [11]. When an individual receives a diagnosis of diabetes they will be faced with marked readjustment of daily life in relation to routine and diet, they may experience worry and stress in their ability to manage the illness and may also feel at odds or frustrated with close family and friends at times. In turn, the emotional strain of adjustment, driven by negative illness appraisals, can result in maladaptive coping behaviours and subsequently result in poorer illness outcomes i.e. blood glucose control, [9]. Diabetes distress therefore explains the connection between emotions, coping strategies and illness outcomes in diabetes, [12].

Diabetes distress is a rational response to the demands of a long-term illness and it should not be perceived as a 'condition' in the same way that clinical depression may be. In an effort to elucidate the confusion between depression and diabetes distress, Fisher and colleagues have suggested that emotional distress should be understood as existing on a continuum, and is represented in terms of "magnitude" or severity, and the "content" or nature of the distress [13]. "Content" refers to those factors attributed to the feelings of distress i.e. diet control, strain on social relationships, feeling overwhelmed by the diabetes, and severity relates to the extent to which an individual experiences these feelings of distress- also an indicator of whether a person is experiencing diabetes distress, or whether they are exhibiting symptoms of depression (which does not imply aetiology and is diagnosed when a particular set of symptoms are present), [13]. It is acknowledged that there may be 
a potential overlap between depressive symptoms and diabetes distress to a certain extent, for instance having elevated diabetes distress over time could be a precursor for depression and likewise experiencing depressive symptoms may also elicit diabetes related distress, [14]. To reiterate, diabetes distress and depression can exist separately or co-occur- but the key point is they are different and should be treated as such. By addressing the aetiology and the severity of the distress as proposed by Fisher and colleagues, one can begin to distinguish which an individual is likely to be experiencing, and take the appropriate action from there, [13]. In day to day practice, health professionals should be encouraged to address the more concealed aspects of wellbeing such as emotional state, as well as adhering to the standard medical protocol. Taking the example of general practitioner appointments; time and attention should not be restricted to the somatic concerns of diabetes, as often these may be related to an underlying psychological or behavioural cause, which may indicate diabetes distress and which could possibly be accessed by simply asking a person with diabetes how they are coping.

\section{Formal measures of Diabetes Distress and Clinical Applications}

Two scales are accepted as statistically sound measures of diabetes distress; The Problem Areas in Diabetes scale (PAID), [15,16] and the Diabetes Distress Scale (DDS), [11]. The PAID scale was the first to offer a more comprehensive psychometric tool to identify the specific psychological difficulties experienced by individuals with diabetes. It is a 20-item questionnaire that uses a 6-point scale to measure the response to common negative emotions and thoughts in relation to living with diabetes, [15]. PAID scores are associated with glycaemic control, self-care behaviour [15, 17] and can be used to track changes in diabetes distress overtime, [18]. Five item and single item versions of PAID have been developed as brief screening tools and are comparably valid and reliable to the full PAID, [19]. Despite its strengths, the PAID scale does not contain any questions addressing an individual with diabetes' experience of the health practitioners involved in their care, it does not use subscales to distinguish the various elements of diabetes distress, and experts have suggested that some of the questions included may be difficult for patients to interpret and understand, [11]. In response to these criticisms, the developers of the PAID scale developed a 17-item questionnaire to measure diabetes distress, the Diabetes Distress Scale-17 (DDS-17), [11]. The tool was developed in collaboration with individuals with diabetes themselves in order to identify which questions from existing diabetes-related psychometric scales were the most appropriate, and to omit any questions deemed irrelevant or uncomprehensive. The DDS-17 distinguishes four main subtypes from the principal term which includes; physician-related distress e.g. "Feeling that my doctor does not give me clear enough directions on how to manage my diabetes", emotional burden e.g. "Feeling angry, scared and/or depressed when I think about living with diabetes", interpersonal distress e.g. "Feeling that friends or family don 't appreciate how difficult living with diabetes can be", and distress related to the diabetes regimen e.g. "Feeling I am not testing my blood sugars frequently enough", [11]. Note that the example questions above tap into emotions or mood, however they are also attached to specific aspects of diabetes coping and adjustment, thus are contextually bound. The DDS-17 displays excellent internal consistency indicating that the component questions are measuring a similar construct, [11] and like PAID there is also a brief version; the DDS-2, [20].

High scores i.e. scores above 40 on PAID [15] and above 3 on the DDS [11] indicate elevated diabetes distress, and have been found to significantly correlate with the presentation of poorer lifestyle (diet control and exercise) and higher $\mathrm{HbA}_{1 \mathrm{c}}$ (measure of average plasma glucose over 3 months), [21]. Diabetes Distress, but not clinical depression, has been consistently found to be significantly associated with elevated $\mathrm{HbA}_{1 \mathrm{c}}$ in individuals with type 2 diabetes $[1,22,23]$ which has been demonstrated across various populations and cultures, [24-28]. The higher the diabetes distress is, the greater the impact will be on diabetes management. At present there is no preferred choice of 
scale in the literature, and although both have evident strengths, one scale needs to be widely accepted as the standard measure if further progress is to be made in the field i.e. presenting all health professionals with a standardised formal screening tool for diabetes distress. These scales are helpful as they provide a standardized way of assessing the level of emotional distress an individual is experiencing, and the DDS-17 is particularly beneficial for physicians as it can be used to distinguish in which of the four domains a person is most strongly affected so that this issue can be more directly addressed. With an awareness of how to recognize diabetes distress, clinicians can use the DDS-17 or PAID scale to identify diabetes distress. Items contained in the scales can provide physicians with a guide for discussing diabetes-related emotional problems during appointments.

\section{Prevalence}

Diabetes distress is common and worldwide may affect as many as $40 \%$ of people diagnosed with diabetes, [29]. Diabetes distress, among other affective disorders has been shown to increase over time in individuals with type 2 diabetes relative to controls, [20]. In the DAWN2 study (Diabetes Attitudes, Wishes and Needs: second study), rates and experiences of diabetes distress among family members of people with diabetes vary, which implies cross-cultural variation; though this may be partially due to the use of different versions of the original scales to measure diabetes distress [29].

\section{Psychosocial Aspects of Diabetes Distress}

The most extensive investigation into the psychosocial aspects of diabetes is the DAWN2 study (Diabetes Attitudes, Wishes and Needs: Second Study), [29]. DAWN2 is a cutting-edge exploration of how family members of people with diabetes across various countries experienced the psychosocial impact of diabetes. To access these experiences participants were required to complete a questionnaire verbally by phone or in person or online via a computer. Using a battery of psychosocial measures the study demonstrated that just over a third of all family members regarded supporting self-care to be burdensome and over half of participants reported feeling frequently worried about medical complications. Importantly, the project revealed that a substantial number of participants were not aware of how they could support their relative with diabetes and many wished to have more of a role in self-management, (39.4\%). DAWN2 offers a database of psychometric screening tools for diabetes distress in families of individuals with diabetes and provides important benchmarks for improving support for those with diabetes and their families in order to more positively manage diabetes. It is a progressive milestone in the literature and ramifies the importance of interpersonal factors and social context in diabetes care.

Perceived support, along with the appraisal of available coping resources, is hugely influential in psychological adjustment, [30]. Though, so far no studies have examined each of the four subdomains of social support i.e. Information-based, emotion-based, affirmation and tangible in the context of self-management and diabetes distress [30]. Protectiveness in partners of individuals with diabetes has also been found to influence physical outcomes and coping (Johnson et al 2014). Johnson and others found overprotectiveness to be significantly associated with more adverse self-management behaviours, with level of diabetes distress mediating this association when levels of physical activity are reduced, [31]. Though, the generalizability of these findings is questionable due to the recruitment of mainly white, middle-class couples from a diabetes clinic in Mid-Western US. Self-efficacy is also demonstrated to have mediating effects on the association between high levels of protectiveness in partners, and people with diabetes' rating of diabetes distress [32]. Working on increasing selfefficacy is a common feature of interventions designed to improve psychological wellbeing and outcomes in diabetes, particularly in relation to enhancing personal control. 


\section{Formal Intervention Approaches}

The most widely available and practiced interventions in diabetes are structured diabetes education groups. Positive self-care and coping strategies have an integral role in preventing diabetes complications, which is the rationale behind structured diabetes education and self-management groups. Structured diabetes education aims to provide individuals who have received a diagnosis of diabetes with the relevant information in order to better understand and manage their illness, [38]. Although the groups have been shown to be helpful for individuals with diabetes and their families, the rate of attendance is generally poor, [29] and effectiveness on HbA1c and quality of life is usually short-term [39-41]. Structured education has been shown to work best when it includes motivational work and goal setting. This is evidenced in the Irish DAFNE (Dose Adjustment for Normal Eating) study, a mixed method RCT (randomized controlled trial) which demonstrated a reduction in $\mathrm{HbA}_{1 \mathrm{c}}$ and an increase in positive self-care behaviours at 12 months follow-up, [42]. Having also used interviews, the authors were able to identify in more depth which aspects of the programme were most effective. This included self-efficacy, worries about diet control and feeling helpless even with the guidance provided by DAFNE, which corresponds strongly with those aspects of emotional distress proposed by the diabetes distress model. This reinstates the importance of considering the model in intervention strategies. A key issue with many, if not all diabetes education programmes is the lack of emphasis on the relevant emotional struggles experienced by individuals and their families. At present, the majority of psychological interventions in diabetes are aimed at alleviating clinical comorbidities in diabetes, such as depression and anxiety, and even they are scarcely made available to people with diabetes, [6]. While treatments in depression are shown to improve mood, there is little evidence to suggest that treating depression also reduces $\mathrm{HbA}_{1 \mathrm{c}}$, or any other parameters clinically relevant to diabetes management, [43, 44]. Though, in a study using Cognitive Behavioural Therapy for Adherence and Depression (CBT-AD) in Type 2 Diabetes, (a treatment which combined both nonadherence and depression interventions and which was delivered by a multi-disciplinary team i.e. dietician, psychologist and a diabetes nurse), depression was reduced, self-management behaviours improved and $75 \%$ of participants had better blood glucose control post-treatment, [45]. But perhaps this is related to the integrative nature of the intervention, which incorporated psychological, behavioural and educational strategies, rather than simply focusing solely on an individual's mood. Psychological therapies (i.e. psychotherapy, counselling) applied in the context of diabetes are largely aimed at reducing emotional distress, depression and anxiety, but they do not address the practical elements of diabetes self-care and therefore they do not improve HbA1c, [46, 47]. The clinical relevance of these approaches in improving diabetes outcomes is therefore dubious.

An intervention study called REDEEM was recently piloted in an attempt to address diabetes distress pragmatically. Individuals with Type 2 diabetes partook in a computerised intervention programme focusing on each of the four sub-domains proposed by the DDS-17 [11], and the findings indicated a significant reduction in diabetes distress and an increase in positive self-care behaviours after the programme was completed, [48]. This reduction was particularly evident in participants who scored highest on regime-related distress at baseline, implying the need for intervention at the earliest possible stage, [48]. Despite these results REDEEM did not reduce $\mathrm{HbA}_{1 c}$, which implies the need to address diabetes distress holistically and in a way that is more applicable to everyday life. The REDEEM trial is not evidence-based and therefore only targets diabetes distress at surface-level, omitting important psychosocial factors such as conflicts with family members and perceived support. It also excludes those unable or unwilling to use computing devices, which demonstrates the importance of looking at the intervention preferences of individuals with diabetes themselves in order to better affix attendance. 
Interventions found to be effective in reducing diabetes distress as well as improving $\mathrm{HbA}_{1 \mathrm{c}}$ are those adopting a psycho-educational stance. A recent RCT, PRIMAS (Programme for diabetes education and treatment for a self-determined living with type 1 diabetes); a new education project incorporating Motivational Interviewing techniques, was compared to an existing structured education programme (Diabetes Teaching and Treatment Programme: DTTP), [49]. PRIMAS, which expanded on each of the original DTTP sessions by implementing the usual education-based material with sessions on goal setting, self-motivation, diabetes distress and sessions for families/ partners, achieved a significant reduction in $\mathrm{HbA1c}$ and diabetes distress in comparison to the DTTP control group at 6 months follow up. No difference was seen between the two groups in depression score, which is not surprising as the programme was not primarily designed to target depressive symptoms. PRIMAS demonstrates that integrating cognitive, emotional and interpersonal factors into diabetes education is more effective in addressing the unseen struggles experienced by individuals with diabetes along with their families. Another pilot study compared the effectiveness of a programme incorporating coping skills training (following cognitive behavioural therapy techniques) and diabetes self-management training, with a standard diabetes education and intervention programme, [50]. Results demonstrated an improvement in systolic blood pressure, cholesterol and $\mathrm{HbA1c}$ and a reduction in diabetes distress at 3 months, which held when retested at 12 and 24 months.

\section{Limitations and Future Directions}

A key issue with the existing literature on diabetes distress is the lack of generalizability to countries outside North America, particularly in relation to the UK and European countries. Very few studies are based in the UK, Ireland and Europe and there are enormous differences in relation to healthcare systems and other social and economic variables in comparison. Research needs to pervade these geographical borders to establish the relevance of the original definition of diabetes distress and the prevalence and psychosocial impact of diabetes distress, particularly in understudied regions such as the UK. It is imperative this occurs in order to design a cross-culturally applicable, evidence-based intervention addressing the specific psychosocial aspects of diabetes distress [29]. Another concern with existing intervention trials is that the majority of participants recruited achieved only subthreshold levels of diabetes distress, meaning that they were not experiencing elevated diabetes distress to begin with. This cautions the validity of significant reductions in diabetes distress. It is important that intervention trials target those people with diabetes who reach the threshold for elevated distress at baseline to better assess the effectiveness of the programme delivered. Moreover, many of the previously piloted trials do not adhere fully to the MRC (Medical Research Council) guidelines by developing a relevant evidence base and a theoretical underpinning to model the intervention on, which are important requirements for feasibility testing, [51]. Many interventions have not taken into account views of individuals with diabetes themselves with regards to what the intervention should entail, and instead interventions have been based on using pre-existing methods such as cognitive behavioural therapy, which is not specifically designed to address diabetes-related emotional problems. A final observation is that the majority of interventions are not always inclusive of partners or carers in the programme, which, as discussed is an integral part of psychological adjustment and should thus be an important focus for improving diabetes outcomes.

To recap, intervention strategies should have a strong theoretical foundation, combining all relevant psychological and interpersonal factors with diabetes self-care education in order to address all of aspects of adjustment in unison, [52]. While these interventions will not have a primary effect on $\mathrm{HbA1c}$, they aim to address the psychological constructs underpinning emotional distress in order to improve self-care, which, in turn will benefit physical outcomes in the long-term, [53]. Crucially, the design of a feasible and effective intervention that is integrative in its approach should be designed in 
collaboration with individuals with diabetes themselves and their significant other, so that it is conceptually driven, person-centred and informed by the people who are at the core of the intervention. For this reason, further research into intervention preferences would greatly subsidise existing literature on diabetes distress. Regarding the facilitation of intervention programmes, incorporating psychologists in a general diabetes care may endorse a more collaborative approach towards diabetes, and this may also reduce any anxiety experienced by health professionals in delivering the psychological and cognitive phases of interventions, [54].

\section{Informal ways to Approach Diabetes Distress: The Power of Conversation}

Even with the establishment of formal measures of diabetes distress, there are strong merits of simply talking to an individual about how they are coping with and feeling about various aspects of their diabetes in order to identify emotional distress. This is common sense, though there is a tendency to believe that discussion of psychological issues is outside of the domains of medical healthcare and time restrictions are also a major obstacle in facilitating such discussions. General medical practitioners may feel they should prioritise somatic concerns over all other non-medical issues, but according to what has been so far established in the literature, ignoring emotional problems may impact negatively on physical wellbeing due to the neglect of self-care and inability to effectively cope, [30,53]. Beverly and colleagues explored communication between people with diabetes and their doctor and the impact of engaging or not engaging in discussions relating to diabetes self-care, [55]. They found that individuals with diabetes who were less likely to discuss self-care issues (30\% of entire sample) reported higher diabetes distress, poorer quality of life, less frequent selfmanagement behaviours and less self-motivated coping strategies [55]. The authors also suggest that individuals who need support the most are potentially going to be the least willing to engage with their doctor or nurse, which underpins the importance of prompting discussion during appointments to present people with an opportunity to talk about which aspects of diabetes care are the most difficult. This does not require psychological expertise and does not require providing solutions there and then. It is simply about initiating normal conversation, listening to and showing an understanding and awareness of the psychosocial and emotional issues embedded in diabetes, which often go unnoticed and grow. As previously indicated, the four subdomains of diabetes distress provide a helpful framework to guide conversations with individuals struggling to manage their diabetes. Selfempowerment is a huge part of coping; however individuals struggling to cope should be made aware that adjustment to diabetes is a gradual process, with many peaks and troughs. Health workers should encourage people with diabetes to feel empowered by regaining a sense of ownership and control over their care plan; while also feeling suitably supported from external sources when need be, [4].

\section{Closing Message}

Diabetes distress is still a relatively novel concept and much work is required to assess its crosscultural applicability, and clinical value in improving $\mathrm{HbA}_{1 \mathrm{c}}$ in groups experiencing elevated diabetes distress. Interventions aimed at reducing diabetes distress should adopt an integrated approach, but most importantly in order to specifically target those factors most strongly related to diabetes distress they have to be modelled on evidence. The intervention should be person-centred and thus designed at grass-root level i.e. informed by individuals with diabetes and their families, which will account also for cultural and contextual specificity. Above all, it is crucial that health practitioners increase their awareness of what diabetes distress is, to understand it as a normal aspect of adaption to a very demanding illness, and to integrate these struggles into everyday diabetes talk. 


\section{References}

1. Fisher, L., Mullan, J. T., Arean, P., Glasgow, R. E., Hessler, D., \& Masharani, U. (2010). Diabetes distress but not clinical depression or depressive symptoms is associated with glycemic control in both cross-sectional and longitudinal analyses. Diabetes Care, 33(1), 23 28.

2. Gale, E. A. (2002). The rise of childhood type 1 diabetes in the 20th century.Diabetes, 51(12), 3353-3361.

3. Holden, S. E., Gale, E. A., Jenkins-Jones, S., \& Currie, C. J. (2014). How many people inject insulin? UK estimates from 1991 to 2010. Diabetes, Obesity and Metabolism, 16(6), 553-559.

4. Ramsay Wan, C., Vo, L., \& Barnes, C. S. (2012). Conceptualizations of patient empowerment among individuals seeking treatment for diabetes mellitus in an urban, public-sector clinic. Patient education and counseling, 87(3), 402-404.

5. Roy, T., \& Lloyd, C. E. (2012). Epidemiology of depression and diabetes: a systematic review. Journal of affective disorders, 142, S8-S21.

6. Diabetes, U. K. (2008). Minding the Gap: The provision of psychological support and care for people with diabetes in the UK. Diabetes UK, London. [Accessed 21/11/2014.]

7. Subramanian, D. N., \& Hopayian, K. (2008). An audit of the first year of screening for depression in patients with diabetes and ischaemic heart disease under the Quality and Outcomes Framework. Quality in primary care, 16(5), 341-344.

8. Abu-Roomi, S. R., Mallipedhi, A., Bain, S. C., Price, D. E., \& Stephens, J. W. (2012). Quality and Outcomes Framework screening questions for depression in patients with diabetes: effective but non-efficient. Diabetic Medicine, 29(7), 957-958.

9. Fisher, L., Skaff, M. M., Mullan, J. T., Arean, P., Mohr, D., Masharani, U., ... \& Laurencin, G. (2007). Clinical depression versus distress among patients with type 2 diabetes not just a question of semantics. Diabetes Care, 30(3), 542-548.

10. Fisher, L., Hessler, D. M., Polonsky, W. H., \& Mullan, J. (2012). When is diabetes distress clinically meaningful? Establishing cut points for the Diabetes Distress Scale. Diabetes Care, 35(2), 259-264.

11. Polonsky, W.H., Fisher, L., Earles, J., et al. (2005). Assessing Psychosocial Distress in Diabetes. Diabetes Care, 28(3), 626-631.

12. Jones, A., Vallis, M., \& Pouwer, F. (2014). If it does not significantly change HbA1c levels why should we waste time on it? A plea for the prioritization of psychological well-being in people with diabetes. Diabetic Medicine.

13. Fisher, L., Gonzalez, J. S., \& Polonsky, W. H. (2014). The confusing tale of depression and distress in patients with diabetes: a call for greater clarity and precision. Diabetic Medicine, 31(7), 764-772.

14. Hosoya, T., Matsushima, M., Nukariya, K., \& Utsunomiya, K. (2011). The relationship between the severity of depressive symptoms and diabetes-related emotional distress in patients with type 2 diabetes. Internal medicine (Tokyo, Japan), 51(3), 263-269.

15. Polonsky WH, Jacobson A, Anderson B et al (1995) Assessment of diabetes-related-distress. Diabetes Care, 18:754-760

16. Welch, G. W., Jacobson, A. M., \& Polonsky, W. H. (1997). The Problem Areas in Diabetes Scale: an evaluation of its clinical utility. Diabetes care, 20(5), 760-766.

17. Snoek, F. J., Pouwer, F. R. A. N. Ç. O. I. S., Welch, G. W., \& Polonsky, W. H. (2000). Diabetes-related emotional distress in Dutch and US diabetic patients: cross-cultural validity of the problem areas in diabetes scale. Diabetes Care, 23(9), 1305-1309. 
18. Welch, G., Weinger, K., Anderson, B., \& Polonsky, W. H. (2003). Responsiveness of the problem areas in diabetes (PAID) questionnaire.Diabetic Medicine, 20(1), 69-72.

19. McGuire, B. E., Morrison, T. G., Hermanns, N., Skovlund, S., Eldrup, E., Gagliardino, J., Kokoszka, A., Matthews, D., Pibernik-Okanović, M., Rodríguez-Saldaña, J., de Wit, M., Snoek, F.J. (2010). Short-form measures of diabetes-related emotional distress: the Problem Areas in Diabetes Scale (PAID)-5 and PAID-1. Diabetologia, 53(1), 66-69.

20. Fisher, L., Glasgow, R. E., Mullan, J. T., Skaff, M. M., \& Polonsky, W. H. (2008). Development of a brief diabetes distress screening instrument. The Annals of Family Medicine, 6(3), 246-252.

21. Graue, M., Haugstvedt, A., Wentzel-Larsen, T., Iversen, M. M., Karlsen, B., \& Rokne, B. (2012). Diabetes-related emotional distress in adults: reliability and validity of the Norwegian versions of the Problem Areas in Diabetes Scale (PAID) and the Diabetes Distress Scale (DDS). International journal of nursing studies, 49(2), 174-182.

22. Fisher, L., Skaff, M. M., Mullan, J. T., Arean, P., Glasgow, R., \& Masharani, U. (2008). A longitudinal study of affective and anxiety disorders, depressive affect and diabetes distress in adults with type 2 diabetes. Diabetic Medicine, 25(9), 1096-1101.

23. Fisher, L., Glasgow, R. E., \& Strycker, L. A. (2010). The relationship between diabetes distress and clinical depression with glycemic control among patients with type 2 diabetes. Diabetes Care, 33(5), 1034-1036.

24. Hayashino, Y., Okamura, S., Matsunaga, S., Tsujii, S., \& Ishii, H. (2012). The association between problem areas in diabetes scale scores and glycemic control is modified by types of diabetes therapy: diabetes distress and care registry in Tenri (DDCRT 2). Diabetes research and clinical practice, 97(3), 405-410.

25. Tol, A., Baghbanian, A., Sharifirad, G., Shojaeizadeh, D., Eslami, A., Alhani, F., \& Tehrani, M. M. (2012). Assessment of diabetic distress and disease related factors in patients with type 2 diabetes in Isfahan: A way to tailor an effective intervention planning in Isfahan-Iran. $J$ Diabetes Metab Disord, 11:20.

26. Spencer, M. S., Kieffer, E. C., Sinco, B. R., Palmisano, G., Guzman, J. R., James, S. A., Graddy-Dansby, G., Two Feathers, J., \& Heisler, M. (2006). Diabetes-specific emotional distress among African Americans and Hispanics with type 2 diabetes. Journal of health care for the poor and underserved, 17(2), 88-105.

27. Sasi, S. T., KoDali, M., Burra, K. C., Muppala, B. S., Gutta, P., \& Bethanbhatla, M. K. (2013). Self Care Activities, Diabetic Distress and other Factors which Affected the Glycaemic Control in a Tertiary Care Teaching Hospital in South India. Journal of clinical and diagnostic research: JCDR, 7(5), 857.

28. Kogan, S. M., Brody, G. H., \& Chen, Y. F. (2009). Depressive symptomatology mediates the effect of socioeconomic disadvantage on $\mathrm{HbA}<\mathrm{sub}>1 \mathrm{c}</$ sub $>$ among rural African Americans with type 2 diabetes. Journal of psychosomatic research, 67(4), 289-296.

29. Nicolucci, A., Kovacs Burns, K., Holt, R. I. G., Comaschi, M., Hermanns, N., Ishii, H., Kokoszka, A., Pouwer, F., Skovlund, S.E., Stuckey, H., Tarkun, I., Vallis, M., Wens, J., Peyrot, M. (2013). Diabetes Attitudes, Wishes and Needs second study (DAWN2TM): Crossnational benchmarking of diabetes-related psychosocial outcomes for people with diabetes. Diabetic Medicine, 30(7), 767-777.

30. Karlsen, B., \& Bru, E. (2014). The relationship between diabetes-related distress and clinical variables and perceived support among adults with type 2 diabetes: A prospective study. International journal of nursing studies, 51(3), 438-447. 
31. Johnson, M. D., Anderson, J. R., Walker, A., Wilcox, A., Lewis, V. L., \& Robbins, D. C. (2014). Spousal overprotection is indirectly associated with poorer dietary adherence for patients with type 2 diabetes via diabetes distress when active engagement is low. British journal of health psychology.

32. Schokker, M. C., Links, T. P., Bouma, J., Keers, J. C., Sanderman, R., Wolffenbuttel, B. H., $\&$ Hagedoorn, M. (2011). The role of overprotection by the partner in coping with diabetes: A moderated mediation model. Psychology and Health, 26(1), 95-111.

33. Rutten, G. E. H. M. (2005). Diabetes patient education: time for a new era.Diabetic Medicine, 22(6), 671-673.

34. Dube, L., Van den Broucke, S., Housiaux, M., Dhoore, W., \& Rendall-Mkosi, K. (2014). Type 2 Diabetes Self-management Education Programs in High and Low Mortality Developing Countries A Systematic Review. The Diabetes Educator. [DOI: 0145721714558305.]

35. Davies, M. J., Heller, S., Skinner, T. C., Campbell, M. J., Carey, M. E., Cradock, S., et al. (2008). Effectiveness of the diabetes education and self management for ongoing and newly diagnosed (DESMOND) programme for people with newly diagnosed type 2 diabetes: cluster randomised controlled trial. British Medical Journal, 336(7642), 491-495.

36. Norris, S. L., Engelgau, M. M., \& Narayan, K. V. (2001). Effectiveness of self-management training in type 2 diabetes a systematic review of randomized controlled trials. Diabetes care, 24(3), 561-587.

37. Casey, D., O'Hara, M. C., Meehan, B., Byrne, M., Dinneen, S. F., \& Murphy, K. (2014). A Mixed Methods Study Exploring the Factors and Behaviors That Affect Glycemic Control Following a Structured Education Program: The Irish DAFNE Study. Journal of Mixed Methods Research, 1558689814547579.

38. Markowitz, S. M., Gonzalez, J. S., Wilkinson, J. L., \& Safren, S. A. (2011). A review of treating depression in diabetes: emerging findings. Psychosomatics, 52(1), 1-18.

39. Lustman, P. J., Anderson, R. J., Freedland, K. E., De Groot, M., Carney, R. M., \& Clouse, R. E. (2000). Depression and poor glycemic control: a meta-analytic review of the literature. Diabetes care, 23(7), 934-942.

40. Gonzalez, J. S., \& McCarl, L. A. (2010). Cognitive behavioral therapy for adherence and depression (CBT-AD) in type 2 diabetes. Journal of cognitive psychotherapy, 24(4), 329.

41. Simson, U., Nawarotzky, U., Friese, G., Porck, W., Schottenfeld-Naor, Y., Hahn, S., et al (2008). Psychotherapy intervention to reduce depressive symptoms in patients with diabetic foot syndrome. Diabetic Medicine, 25(2), 206-212.

42. Shibayama, T., Kobayashi, K., Takano, A., Kadowaki, T., \& Kazuma, K. (2007). Effectiveness of lifestyle counseling by certified expert nurse of Japan for non-insulin-treated diabetic outpatients: a 1-year randomized controlled trial. Diabetes research and clinical practice, 76(2), 265-268.

43. Fisher, L., Hessler, D., Glasgow, R. E., Arean, P. A., Masharani, U., Naranjo, D., \& Strycker, L. A. (2013). REDEEM: a pragmatic trial to reduce diabetes distress. Diabetes Care, 36(9), 2551-2558.

44. Hermanns, N., Kulzer, B., Ehrmann, D., Bergis-Jurgan, N., \& Haak, T. (2013). The effect of a diabetes education programme (PRIMAS) for people with type 1 diabetes: results of a randomized trial. Diabetes research and clinical practice, 102(3), 149-157.

45. Melkus, G. D. E., Chyun, D., Vorderstrasse, A., Newlin, K., Jefferson, V., \& Langerman, S. (2010). The effect of a diabetes education, coping skills training, and care intervention on physiological and psychosocial outcomes in black women with type 2 diabetes. Biological research for nursing, 12(1), 7-19. 
46. Medical Research Council. A framework for the development and evaluation of RCTs for complex interventions to improve health London: MRC, 2000. [Retrieved from: http://www.mrc.ac.uk/documents/pdf/complex-interventions-guidance/].

47. Peyrot, M., \& Rubin, R. R. (2007). Behavioral and Psychosocial Interventions in Diabetes A conceptual review. Diabetes Care, 30(10), 2433-2440.

48. Karlsen, B., Oftedal, B., \& Bru, E. (2012). The relationship between clinical indicators, coping styles, perceived support and diabetes-related distress among adults with type 2 diabetes. Journal of advanced nursing, 68(2), 391-401.

49. Peyrot, M., Rubin, R. R., Lauritzen, T., Snoek, F. J., Matthews, D. R., \& Skovlund, S. E. (2005). Psychosocial problems and barriers to improved diabetes management: results of the Cross-National Diabetes Attitudes, Wishes and Needs (DAWN) Study. Diabetic medicine, 22(10), 1379-1385.

50. Beverly, E. A., Ganda, O. P., Ritholz, M. D., Lee, Y., Brooks, K. M., Lewis-Schroeder, N. F., Hirose, M., \& Weinger, K. (2012). Look Who's (Not) Talking Diabetic patients' willingness to discuss self-care with physicians. Diabetes care, 35(7), 1466-1472. 